\title{
Safety and feasibility of laparoscopy-assisted distal gastrectomy with suprapancreatic nodal dissection for clinical stage I gastric cancer: a multicenter phase II trial (JCOG 0703)
}

\author{
Hitoshi Katai ${ }^{1}$, Mitsuru Sasako ${ }^{2}$, Haruhiko Fukuda ${ }^{3}$, Kenichi Nakamura ${ }^{3}$, Naoki Hiki $^{4}$, Makoto Saka ${ }^{1}$, \\ Hiroki Yamaue ${ }^{5}$, Takaki Yoshikawa ${ }^{6}$, Kazuyuki Kojima ${ }^{7}$, and the JCOG Gastric Cancer Surgical Study Group \\ ${ }^{1}$ Gastric Surgery Division, National Cancer Center Hospital, 5-1-1 Tsukiji, Chuo-ku, Tokyo 104-0045, Japan \\ ${ }^{2}$ Department of Surgery, Hyogo College of Medicine, Hyogo, Japan \\ ${ }^{3}$ Center for Cancer Control and Information Services, National Cancer Center, Tokyo, Japan \\ ${ }^{4}$ Department of Gastroenterological Surgery, Cancer Institute Hospital, Tokyo, Japan \\ ${ }^{5}$ Second Department of Surgery, Wakayama Medical University, Wakayama, Japan \\ ${ }^{6}$ Department of Gastrointestinal Surgery, Kanagawa Cancer Center, Yokohama, Japan \\ ${ }^{7}$ Department of Esophagogastric Surgery, Tokyo Medical and Dental University, Tokyo, Japan
}

\begin{abstract}
Background. Although the number of patients undergoing laparoscopy-assisted distal gastrectomy (LADG) has been increasing, a prospective study with a sample size sufficient to investigate the benefit of LADG has never been reported. We conducted a multi-institutional phase II trial to evaluate the safety of LADG with nodal dissection for clinical stage I gastric cancer patients.

Methods. The subjects comprised patients with clinical stage I gastric cancer who were able to undergo a distal gastrectomy. LADG with D1 plus suprapancreatic node dissection was performed. The primary endpoint was the proportion of patients who developed either anastomotic leakage or a pancreatic fistula. The secondary endpoints included surgical morbidity and short-term clinical outcome.

Results. Between November 2007 and September 2008, 176 eligible patients were enrolled. The proportion of patients who developed anastomotic leakage or a pancreatic fistula was $1.7 \%$. The overall proportion of in-hospital grade 3 or 4 adverse events was $5.1 \%$. The short-term clinical outcomes were as follows: $\mathbf{4 3 . 2} \%$ of the patients requested an analgesic on postoperative days 5-10; the median time from surgery until the first episode of flatus was 2 days; and 88 patients $(50.0 \%)$ had a body temperature of $38^{\circ} \mathrm{C}$ or higher during their hospital stay.

Conclusions. This trial confirmed the safety of LADG performed by credentialed surgeons in terms of the incidence of anastomotic leakage or pancreatic fistula formation. A phase III trial (JCOG 0912) to confirm the noninferiority of LADG to an open gastrectomy in terms of overall survival is ongoing.
\end{abstract}

Key words Gastric cancer - Laparoscopy-assisted distal gastrectomy $\cdot$ Morbidity

Offprint requests to: $\mathrm{H}$. Katai

Received: May 4, 2010 / Accepted: July 3, 2010

\section{Introduction}

The proportion of patients with early gastric cancer among all gastric cancer patients has increased to more than $50 \%$ at major institutions in Japan [1]. The Japanese guidelines allow laparoscopy-assisted gastrectomy as an investigational treatment for early gastric cancer, with consideration of the patient's performance status [2]. Since Kitano et al. [3] reported the first laparoscopyassisted gastrectomy in 1994, this technique has attracted the attention of surgeons. A nationwide survey of laparoscopic surgery for gastric cancer has shown that the total number of patients who were treated using a laparoscopic technique has increased and that this increase was most remarkable among patients with cT1N0 (stage IA), cT1N1 (stage IB), and cT2N0 (stage IB) tumors [4].

D1 + suprapancreatic node dissection for stage I tumors could indeed be considered as excessive surgery in western countries because of their low incidence of suprapancreatic node metastasis. However, a Japanese database showed that $\mathrm{N} 2$ node metastasis was found in $5.8 \%$ of patients with $\mathrm{T} 1$ tumors (submucosal [SM]) and in $17 \%$ with T2 tumors (muscularis propriae [MP]) [5]. Surgeons should aim at a cure rate of $100 \%$ as a general concept. Therefore, Japanese guidelines demand D1 + suprapancreatic node dissection for this population (i.e., those with clinical stage I gastric cancer).

Recent advances in operative techniques and endoscopic instrumentation have led to the standardization of laparoscopy-assisted distal gastrectomy (LADG) with suprapancreatic node dissection among experienced laparoscopic surgeons. Although whether this modality is appropriate for cancer treatment remains a concern, the technical difficulties of LADG have been 
gradually solved. Several studies have reported that LADG allows a better quality-of-life outcome than an open distal gastrectomy (ODG) [6-11]. Although several studies have reported that LADG is a safe procedure with regard to morbidity and mortality $[12,13]$, other studies have reported that LADG has a higher risk of surgical morbidities such as anastomotic leakage, stenosis, and pancreatic fistula formation than ODG [14, 15]. Therefore, the safety of LADG should first be confirmed as an initial step.

On the other hand, several retrospective and small prospective studies have reported that the long-term results of LADG are similar to those of ODG $[15,16]$. However, a prospective study with a sample size sufficient to investigate the long-term survival of LADG has not yet been reported. Thus, once the safety of LADG has been confirmed, the long-term survival after LADG should be evaluated as a next step.

The present report describes a multi-institutional phase II trial conducted by the Gastric Cancer Surgical Study Group of the Japan Clinical Oncology Group (JCOG 0703) to evaluate the safety of LADG with nodal dissection for clinical stage I gastric cancer patients. If the safety of LADG was verified, a subsequent phase III trial was to be designed to evaluate the noninferiority of LADG to ODG in terms of long-term survival.

\section{Patients and Methods}

Our study was designed as a multicenter, prospective phase II trial. The study protocol was approved by the JCOG Protocol Review Committee and the institutional review boards (IRBs) of each of the 17 participating Japanese hospitals before the activation of the study. The inclusion criteria were as follows: histologically confirmed adenocarcinoma of the stomach, a c-stage IA (T1N0) or IB (T1N1/T2N0) tumor according to the Japanese classification of gastric carcinoma, 2nd English edition [17], no indications for endoscopic mucosal resection (EMR) according to the Japanese endoscopic treatment guidelines [2] ("no indications for EMR" corresponds to a clinical node-positive or clinical nodenegative status with any of the following criteria: a tumor size $2 \mathrm{~cm}$ or larger, invasion to the submucosa or deeper, a histologically undifferentiated type, the presence of an ulcer or ulcerative scar [in the case of depressed type], or the impossibility of an en-bloc resection), a distal gastrectomy-treatable tumor located in the middle or lower third of the stomach, no involvement of the duodenum, a patient age of 20-80 years, an Eastern Cooperative Oncology Group (ECOG) performance status (PS) of 0 or 1, a body mass index (BMI) of less than $30 \mathrm{~kg} / \mathrm{m}^{2}$, the absence of a recurrent tumor after EMR, no prior upper abdominal surgery or intestinal resection other than an appendectomy, no prior chemotherapy or radiotherapy for any malignancy, adequate organ function, and written informed consent. The exclusion criteria were as follows: a synchronous or metachronous (within 5 years) malignancy other than carcinoma in situ or mucosal cancer, pregnancy or lactation, severe mental disease, the systemic administration of corticosteroids, unstable angina or myocardial infarction within 6 months before registration, uncontrolled hypertension, diabetes mellitus (both uncontrolled and controlled with insulin), and severe respiratory disease requiring continuous oxygen therapy.

After the inclusion/exclusion criteria were confirmed by telephoning or faxing the JCOG Data Center, the patients were registered in this JCOG 0703 trial.

\section{Surgery}

An LADG with D1 plus suprapancreatic node dissection was performed. The extent of the suprapancreatic node dissection was decided according to the surgical $\mathrm{T}$ and $\mathrm{N}$ stage of the tumor, based on the second version of the Gastric cancer treatment guidelines in Japan [2]. If the intraoperative findings revealed a tumor stage of II or greater, the LADG was converted to an ODG. Patients requiring a pylorus-preserving distal gastrectomy, but not a total gastrectomy, were included in this series. The size of the minilaparotomy incision was $6 \mathrm{~cm}$ or less, in principle. The reconstruction approach and the surgical method following resection were not specified. Postoperative analgesia, such as the use of epidural anesthesia, also was not specified. Requests for analgesia on postoperative days 5-10 were recorded. Adjuvant chemotherapy with S-1 for 1 year was recommended for patients with a curative resection and a pathological stage II, IIIA, or IIIB tumor.

\section{Follow-up}

All the enrolled patients were followed up at least every 6 months for the first 2 years and then every year for another 3 years until 5 years after the close of registration. Blood tests, an upper gastrointestinal endoscopy, and an abdominal computed tomography examination were performed every year.

\section{Quality control of surgery}

Before the start of this trial, all participating surgeons agreed to the technical details for LADG. Significant experience in gastric cancer surgery, especially in LADG 
and ODG, was a prerequisite for a surgeon's participation in the trial. Only surgeons who had performed 30 or more LADG procedures and 30 or more ODG procedures were selected. Furthermore, we performed a central review of the surgical procedure by photographing all the surgeries and by videotaping arbitrarily selected surgeries. To assess compliance with the lymphadenectomy, the number of dissected nodes at all stations was recorded on the case report forms and the results were monitored.

\section{Endpoints}

The primary study endpoint was the proportion of patients who developed either anastomotic leakage or a pancreatic fistula, defined as the proportion of patients with either a grade 1 or greater anastomotic leakage or a grade 2 or greater pancreatic fistula among all the operated patients. The secondary endpoints were overall survival (OS), relapse-free survival (RFS), the proportion of LADG completions, the proportion of conversions from LADG to ODG, surgical morbidity, and the short-term clinical outcomes. The OS and RFS are not reported in this article because the current follow-up time was not long enough.

In this trial, adverse events were classified based on the Common Terminology Criteria for Adverse Events version 3.0 (CTCAE v 3.0). Anastomotic leakage and pancreatic fistula formation were also categorized based on the CTCAE v 3.0, with the following supplementary explanation of the JCOG surgical morbidity criteria [18]: a grade 1 or more anastomotic leakage was diagnosed radiologically and was recorded regardless of its clinical significance; a grade 2 or greater pancreatic fistula was diagnosed when fluid with a high amylase concentration drained from the peripancreatic area and led to an infection. The completion of LADG was defined as the proportion of patients without conversion from LADG to ODG among all the operated patients. The proportion of conversions from LADG to ODG was defined as the proportion of patients with a conversion among the patients who were diagnosed before gastrectomy as having clinical stage IA or IB. As for both the completion of LADG and the proportion of conversions, all LADG cases that required a skin incision of more than $6 \mathrm{~cm}$ were regarded as conversions to an ODG. The expected values for LADG completions and the proportion of conversions were stated as $70 \%$ and $5 \%$ in the protocol.

The short-term clinical outcomes were: (i) the proportion of patients requesting an analgesic on postoperative days $5-10$, (ii) the time from the end of surgery until the first episode of flatus, (iii) the highest body temperatures during the first 3 days after surgery, and (iv) the highest body temperatures during hospitalization. The expected values for these four endpoints were $20 \%$ or less, within 3 days, $38^{\circ} \mathrm{C}$ or less, and $38^{\circ} \mathrm{C}$ or less, respectively. The data regarding analgesic use were collected on postoperative days $5-10$ because epidural anesthesia was administered on the first 3 or 4 postoperative days in most patients.

\section{Study design and statistical methods}

This trial evaluated the safety of LADG in terms of the incidence of either anastomotic leakage or pancreatic fistula formation. If the incidence of these two postoperative complications was as low as expected, a subsequent phase III trial was designed to evaluate the noninferiority of LADG to ODG in terms of overall survival. In this phase II trial, the sample size was 170 cases, providing a $90 \%$ power under the hypothesis of a primary endpoint with an expected value of $3 \%$ and a threshold value of $8 \%$, using one-sided testing at a $10 \%$ significance level. The expected value was decided according to the postoperative outcome of 1493 patients who had undergone an ODG at institutions belonging to the Gastric Cancer Surgical Study Group in 2004; among these patients, the proportion of those who developed anastomotic leakage was $1.3 \%$ and the proportion of those who developed pancreatic fistula formation was $0.5 \%$. All the statistical analyses were performed using SAS software version 9.1 (SAS Institute, Cary, NC, USA).

This study was registered with UMIN-CTR [www. umin.ac.jp/ctr/], identification number UMIN000000874.

\section{Results}

\section{Patient characteristics}

Between November 2007 and September 2008, 177 patients from 14 out of the 17 hospitals with IRB approval were registered in the trial. One patient was deemed ineligible after enrollment because of a lack of written informed consent, which originated from a miscommunication among the patient's physicians.

The patients' characteristics are summarized in Table 1. The clinical and histological stages in the text are described based on the Japanese classification of gastric carcinoma, 2nd English edition [17], while the results shown in Table 2 are based on both the Japanese classification of gastric carcinoma (hereafter "Japanese classification") and the International Union Against Cancer (UICC) TNM classification, 6th edition. The median age of the patients was 59 years. The male-to-female ratio was nearly $1: 1$. More than $90 \%$ of the patients had clinical 
Table 1. Baseline characteristics

\begin{tabular}{lc}
\hline Age (years) & \\
Median & 59 \\
Range & $24-80$ \\
Sex, no. $(\%)$ & $91(51.7 \%)$ \\
$\quad$ Male & $85(48.3 \%)$ \\
Female & \\
Body-mass index (BMI), no. (\%) & $42(23.9 \%)$ \\
$\quad<20$ & $107(60.8 \%)$ \\
$20-24.9$ & $27(15.3 \%)$ \\
$\geq 25.0$ & \\
Tumor location, no. $(\%)$ & $114(64.8 \%)$ \\
Upper third of stomach & $62(35.2 \%)$ \\
Middle third of stomach & \\
Lower third of stomach & $163(92.6 \%)$ \\
Clinical T stage, no. $(\%)^{\mathrm{a}}$ & $13(7.4 \%)$ \\
T1 & \\
T2 & $175(99.4 \%)$ \\
Clinical node status, no. $(\%)^{\mathrm{a}}$ & $1(0.6 \%)$ \\
N0 & \\
N1 & $162(92.0 \%)$ \\
Clinical stage, no. $(\%)^{\mathrm{a}}$ & $14(8.0 \%)$ \\
IA & \\
IB & \\
\hline a The distributions of c-stage were the same in the Japanese classifica- \\
tion of gastric carcinoma, 2nd English edition [17] and in the Interna- \\
tional Union Against Cancer $($ UICC) TNM classification, 6 th edition
\end{tabular}

stage IA (cT1N0) disease. The median body mass index was 21.8. About two-thirds of the tumors were located in the middle third of the stomach.

The histological findings are shown in Table 2. Early gastric cancer (T1) was confirmed pathologically in about $90 \%$ of the patients. The proportion of patients with nodal involvement was $15.3 \%$ overall (27 out of 176 patients). About $20 \%$ of the node-positive patients had suprapancreatic node metastasis. The accuracy of the preoperative diagnosis of stage I (Japanese classification) disease was $92.6 \%$. Adjuvant chemotherapy with S-1 was performed in 14 patients $(8.0 \%)$.

\section{Operative procedures (Table 3)}

All the operations were performed with curative intent. The median duration of the operations was $250 \mathrm{~min}$. A distal gastrectomy or a pylorus-preserving distal gastrectomy was performed in 174 patients. A total gastrectomy was performed in 2 patients after the identification of a positive left gastroepiploic node and a positive proximal margin, respectively. The proportion of Billroth I operations was about $50 \%$, and this procedure was the most commonly used method.

All the patients underwent a D1 plus some extent of suprapancreatic dissection. Thirty of the 176 patients underwent a D2 lymphadenectomy.

The median length of the skin incision was $5 \mathrm{~cm}$. The length of the skin incision was more than $6 \mathrm{~cm}$ in 5
Table 2. Histological findings

\begin{tabular}{lc}
\hline Histological type, no. (\%) & \\
Differentiated & $69(39.2 \%)$ \\
Undifferentiated & $107(60.8 \%)$ \\
Tumor size (cm) & \\
Median & 2.5 \\
Range & $0.5-10.0$ \\
Histological T stage, no. (\%) (same in the Japanese and \\
UICC classifications) \\
T1 \\
T2 & $156(88.6 \%)$ \\
T3 & $19(10.8 \%)$ \\
Histological node status (Japanese), no. (\%) & $1(0.6 \%)$ \\
N0 & $149(84.7 \%)$ \\
N1 & $22(12.5 \%)$ \\
N2 & $5(2.8 \%)$ \\
Histological stage (Japanese), no. (\%) & \\
IA & $140(79.5 \%)$ \\
IB & $23(13.1 \%)$ \\
II & $9(5.1 \%)$ \\
IIIA & $4(2.3 \%)$ \\
Histological node status (UICC), no. (\%) & \\
N0 & $149(84.7 \%)$ \\
N1 & $25(14.2 \%)$ \\
N2 & $2(1.1 \%)$ \\
Histological stage (UICC), no. (\%) & $140(79.5 \%)$ \\
IA & $22(12.5 \%)$ \\
IB & $14(8.0 \%)$ \\
II &
\end{tabular}

Japanese, Japanese classification of gastric carcinoma, 2nd English edition [17]; UICC, UICC TNM classification, 6th edition

patients. The LADG was successfully completed in 170 (96.6\%; 95\% confidence interval [CI], 92.7-98.7) of the 176 patients, which was far better than the expected value $(70 \%)$. One patient was diagnosed as having stage II disease prior to gastrectomy, and the procedure was converted to an ODG.

The surgical procedure was converted from an LADG to an ODG in 5 patients with a preoperative diagnosis of clinical stage IA or IB; consequently, the proportion of conversions was $2.9 \%$ ( $95 \%$ CI, $0.9 \%-6.5 \%$ ), which was lower than expected. The reasons for conversion were a positive proximal resected margin $(n=1)$, difficulty with the anastomosis $(n=1)$, a skin incision longer than $6 \mathrm{~cm}(n=1)$, and bleeding $(n=2)$.

The median blood loss was $43.5 \mathrm{ml}$, and blood transfusions were required in 3 patients. The median postoperative hospital stay was 12 days (range, 7-58 days).

\section{Operative complications and deaths}

Minor injuries (grade 1, CTCAE v 3.0) were observed in two patients during the operations (transverse colon, right gastroepiploic vein).

Grade 1 or greater anastomotic leakage was observed in two patients. Grade 2 or greater pancreatic fistula formation was observed in two patients. One patient 
Table 3. Details of surgical procedures

\begin{tabular}{|c|c|}
\hline \multicolumn{2}{|l|}{ Operation time (min) } \\
\hline Median & 250 \\
\hline Range & $130-495$ \\
\hline \multicolumn{2}{|l|}{ Type of gastrectomy } \\
\hline Proximal gastrectomy & 0 \\
\hline Pylorus-preserving distal gastrectomy & $43(24.4 \%)$ \\
\hline Distal gastrectomy & $131(74.4 \%)$ \\
\hline Total gastrectomy & $2(1.1 \%)$ \\
\hline \multicolumn{2}{|l|}{ Reconstruction method } \\
\hline Roux-en-Y & $48(27.3 \%)$ \\
\hline Billroth I & $85(48.3 \%)$ \\
\hline Gastro-gastrostomy & $43(24.4 \%)$ \\
\hline \multicolumn{2}{|l|}{ Extent of lymph node dissection, no. (\%) } \\
\hline $\mathrm{D} 1+\alpha^{\mathrm{a}}$ & $4(2.3 \%)$ \\
\hline $\mathrm{D} 1+\beta^{\mathrm{b}}$ & $142(80.7 \%)$ \\
\hline D2 & $30(17.0 \%)$ \\
\hline \multicolumn{2}{|l|}{ Length of skin incision $(\mathrm{cm})$} \\
\hline Median & 5 \\
\hline Range & $3-20$ \\
\hline \multicolumn{2}{|l|}{ Length of skin incision, no. (\%) } \\
\hline$\leq 6 \mathrm{~cm}$ & $171(97.2 \%)$ \\
\hline$>6 \mathrm{~cm}$ & $5(2.8 \%)$ \\
\hline \multicolumn{2}{|l|}{ Number of ports } \\
\hline Median & 5 \\
\hline Range & $4-7$ \\
\hline \multicolumn{2}{|l|}{ Completion of $\mathrm{LADG}^{\mathrm{c}}$} \\
\hline Yes & $170(96.6 \%)$ \\
\hline No & $6(3.4 \%)$ \\
\hline \multicolumn{2}{|l|}{ Conversion to open procedure $(n=175)^{\mathrm{d}}$} \\
\hline Yes & $5(2.9 \%)$ \\
\hline No & $170(97.1 \%)$ \\
\hline \multicolumn{2}{|l|}{ Blood loss (ml) } \\
\hline Median & 43.5 \\
\hline Range & $0-490$ \\
\hline \multicolumn{2}{|l|}{ Perioperative blood transfusion } \\
\hline Yes & $3(1.7 \%)$ \\
\hline No & $173(98.3 \%)$ \\
\hline
\end{tabular}

${ }^{\mathrm{a}} \mathrm{D} 1+\alpha, \mathrm{D} 1$ dissection + dissection of the nodes along the left gastric and common hepatic arteries

${ }^{\mathrm{b}} \mathrm{D} 1+\beta, \mathrm{D} 1+\alpha$ dissection + dissection of the nodes along the celiac artery

${ }^{\mathrm{c}}$ Completion of LADG was counted for all operated patients $(n=176)$

${ }^{\mathrm{d}}$ Conversion to open procedure was counted for all patients diagnosed before gastrectomy as having clinical stage IA or IB

suffered from both an anastomotic leakage and a pancreatic fistula. The proportion of patients with either anastomotic leakage or pancreatic fistula, the primary endpoint, was $1.7 \%(3 / 176 ; 80 \% \mathrm{CI}, 0.6-3.8$; one-sided $P=0.0003$, binomial test of the null hypothesis that the proportion is equal or greater than $8 \%$ ).

The overall proportion of in-hospital adverse events (grade 3 or 4 according to the CTCAE v 3.0) was 5.1\% $(9 / 176)$ and the proportion of grade 1 or greater events (excluding fever) was $9.1 \%$ (16/176). Grade 3 or greater postoperative bleeding, anastomotic stricture, and intestinal obstruction were observed in two patients, one patient, and one patient, respectively.
Re-operations were performed in three patients. The reasons for the re-operations were anastomotic stricture, pancreatic fistula, and obstruction. The patient with the obstruction had developed grade 3 disseminated intravascular coagulopathy (DIC) and required a blood transfusion. The postoperative mortality rate was zero.

\section{Short-term clinical outcomes}

Flatus was recognized in all the patients during the period of hospitalization. The median time from the end of surgery until the first episode of flatus was 2 days (range, 1-5 days).

Seventy-six of the 176 patients $(43.2 \% ; 95 \%$ CI, $35.8-50.9)$ requested an analgesic on postoperative days $5-10$, although we expected this value to be $20 \%$ or lower.

The highest body temperature during the first 3 days after surgery was recorded. The median body temperature during this period was $37.9^{\circ} \mathrm{C}$ (range, $36.6^{\circ} \mathrm{C}-39.1^{\circ} \mathrm{C}$ ). A body temperature of $38^{\circ} \mathrm{C}$ or greater was observed in 67 patients $(38.1 \%)$ on postoperative day 1 , in 38 patients $(21.6 \%)$ on postoperative day 2 , and in 11 patients $(6.3 \%)$ on postoperative day 3 .

The highest body temperature during hospitalization was recorded: 88 patients had a body temperature of $38^{\circ} \mathrm{C}$ or higher during their hospital stay.

\section{Discussion}

The proportion of patients who developed anastomotic leakage or pancreatic fistula formation, the primary endpoint of this study, was $1.7 \%$. The proportion of these two postoperative complications was as low as expected in this study design and was also lower than that reported in previous publications $[14,15]$.

In retrospective reports about LADG, the proportions of patients who developed anastomotic leakage were quite different $(1.7 \%-14 \%)$ [8, 9, 12], while that of patients who developed pancreatic fistula formation was $1.0 \%$ [9]. The primary endpoint in the present prospective study demonstrated that the proportion of patients who developed these two postoperative complications was lower than expected, compared with the proportions in these retrospective reports. In addition, the proportion of patients who developed these complications was considered to be equivalent to that of those who developed complications after ODG, where the proportion of patients who developed anastomotic leakage has been reported as $0.6 \%-2.7 \%$ and that of patients who developed pancreatic fistula as $0.6 \%$ in Japan $[12,19,20]$. The overall proportion of in-hospital adverse events itself was $5.1 \%$, which was also relatively 
low $[12,15]$. These results demonstrated that LADG can be performed very safely by credentialed surgeons.

One possible reason for the low proportion of inhospital complications in the present study might be the strict criteria that were used to select the attending surgeons. We only selected surgeons who had performed more than 30 LADG procedures and more than 30 ODG procedures prior to this trial, because we were aware that LADG requires more experience to obtain sufficient skill than its conventional open counterpart. This low complication rate also showed that the experience of performing 30 LADG procedures with suprapancreatic node dissection was sufficient to achieve proficiency. The strict selection of the attending surgeons might also have contributed to the favorable results regarding the high proportion of successful LADG procedures and the low proportion of conversions to ODG. These rates were low enough to justify a subsequent phase III trial (LADG vs ODG) [7, 21].

A second possible reason for the low proportion of in-hospital complications in the present study might be the measures used to ensure the quality control of the actual surgeries. In addition to our strict criteria for selecting surgeons, we obtained a consensus regarding the details of the surgical procedure prior to the start of the clinical trial, and the procedures were reviewed using photographs and videotapes during the enrollment period.

A third possible reason for the low proportion of inhospital complications in the present study was the low body mass index (BMI) of the registered patients. Patients with a BMI of more than 30 were excluded from this study; as a result, the median BMI was 21.8. In western countries, the median BMI was reported as $26-29$, and higher morbidities $(10 \%-26 \%$ as grade $1-4$ adverse events) than that in this study $(9.1 \%)$ were demonstrated [22, 23]. Thus, BMI should be taken into consideration to interpret the morbidity results. Of note, at the National Cancer Center Hospital, Tokyo, the proportion of patients with a BMI of 25 or more was $24.0 \%$, which is higher than the proportion in this study $(15.3 \%)$. This implies that the surgeons might have preferred nonobese patients for the present trial [24].

The accuracy of preoperative staging is a key factor in the implementation of a subsequent phase III trial. Some surgeons consider that LADG should not be applied for stage II or more advanced disease. From the viewpoint of these surgeons, the accuracy of the preoperative diagnosis needs to be confirmed in this phase II study. The accuracy of the preoperative diagnosis for stage I disease (Japanese classification) was 92.6\%, which was sufficient to convince these surgeons of the feasibility of a subsequent phase III study.

The major advantage of LADG over ODG is that it is less invasive. Therefore, we evaluated the short-term clinical outcomes (proportion of patients who requested an analgesic on postoperative days 5-10, time from end of surgery until first episode of flatus, and body temperature) as secondary endpoints. A visual analog scale is the most common way of evaluating pain [6]. This scale is, however, a subjective indicator and is not appropriate for comparison with historical data in a singlearm setting. Instead, we adopted the proportion of patients who requested an analgesic as a measure of pain. The proportion of patients who requested an analgesic was more than $40 \%$, which was higher than expected and higher than that described in previous reports $[6,7,25]$. This result was paradoxical. Because this study was a phase II study, this endpoint will need to be evaluated further in a subsequent phase III trial (LADG vs ODG).

The time from the end of surgery until the first episode of flatus was a good indicator of bowel function recovery. The time until flatus was as we expected and was similar to the results of a previous report [6].

Measures of inflammation are important for evaluating the invasiveness of surgery. Body temperature is one parameter of inflammation. A body temperature greater than $38^{\circ} \mathrm{C}$ is one of the clinical manifestations of systemic inflammatory response syndrome (SIRS) [26], and patients can suffer greatly from this complication. The change in body temperature was as we expected and was similar to the results of previous reports [27, 28].

Although the safety of LADG was confirmed in the present study, this result may not fit in western countries. LADG will remain an investigational treatment even in Japan until its effectiveness is fully evaluated in a phase III trial. We are now conducting a JCOG phase III trial (JCOG 0912) with a recruitment of about 1000 patients to confirm the noninferiority of LADG to ODG in terms of overall survival.

\section{Conclusion}

The present trial confirmed the safety of LADG performed by credentialed surgeons in terms of the incidence of anastomotic leakage or pancreatic fistula formation. A phase III trial to confirm the noninferiority of LADG to ODG in terms of overall survival is now ongoing.

Acknowledgments We thank Mr. Taro Shibata for the statistical analyses and Ms. Naoko Murata and Ms. Yuka Sakamoto, JCOG Data Center, for the data management. This study was supported in part by Grants-inAid for Cancer Research (17S-3, 17S-5, 17-11, 20S-3, 20S-6) from the Ministry of Health, Labour and Welfare, Japan. 


\section{Appendix}

\section{Study participants}

The following institutions and investigators participated in the trial: Hakodate Goryoukaku Hospital (A. Takagane), Yamagata Prefectural Central Hospital (N. Fukushima), National Cancer Center Hospital ( $\mathrm{H}$. Katai, M. Saka), Tokyo Medical and Dental University (K. Kojima, M. Inokuchi, H. Yamada), Cancer Institute Hospital (N. Hiki, T. Fukunaga, H. Yoshiba, M. Tokunaga), Kanagawa Cancer Center (T. Yoshikawa, H. Cho), Aichi Cancer Center Hospital (Y. Mochizuki, K. Misawa), Fujita Health University (I. Uyama, S. Kanaya, K. Taniguchi), Kinki University Hospital (H. Imamoto), Osaka Medical Center for Cancer and Cardiovascular Diseases (I. Miyashiro), Department of General and Gastroenterological Surgery, Osaka Medical College (N. Tanigawa), Wakayama Medical University (M. Iwahashi, K. Takifuji), Hiroshima City Hospital (M. Nishizaki), and Oita University (S. Kitano, N. Shiraishi, T. Eto).

\section{References}

1. Kakizoe T, Yamaguchi N, Mitsuhashi F, Koshiji M, editors. Cancer statistics in Japan 2001. Tokyo: Foundation for Promotion of Cancer Research; 2001. p. 46-9.

2. Nakajima T. Gastric cancer treatment guidelines in Japan. Gastric Cancer 2002;5:1-5.

3. Kitano S, Iso Y, Moriyama M, Sugimachi K. Laparoscopy-assisted Billroth I gastrectomy. Surg Laparosc Endosc 1994;4:146-8.

4. Etoh T, Shiraishi N, Kitano S. Current trends of laparoscopic gastrectomy for gastric cancer in Japan. Asian J Endosc Surg 2009;2:18-21.

5. Sasako M. Principles of surgical treatment for curable gastric cancer. J Clin Oncol 2003;21:274s-5s.

6. Kitano S, Shiraishi N, Fujii K, Yasuda K, Inomata M, Adachi Y. A randomized controlled trial comparing open vs laparoscopyassisted distal gastrectomy for the treatment of early gastric cancer: an interim report. Surgery 2002;131:S306-11.

7. Kim MC, Kim KH, Kim HH, Jung GJ. Comparison of laparoscopy-assisted by conventional open distal gastrectomy and extraperigastric lymph node dissection in early gastric cancer. J Surg Oncol 2005;91:90-4.

8. Dulucq JL, Wintringer P, Stabilini C, Solinas L, Perissat J, Mahajna A. Laparoscopic and open gastric resections for malignant lesions: a prospective comparative study. Surg Endosc 2005;19:933938.

9. Naka T, Ishikura T, Shibata S, Yamaguchi Y, Ishiguro M, Yurugi E, et al. Laparoscopy-assisted and open distal gastrectomies for early gastric cancer at a general hospital in Japan. Hepatogastroenterology 2005;52:293-7.

10. Kim YW, Baik YH, Yun YH, Nam BH, Kim DH, Choi IJ, et al. Improved quality of life outcomes after laparoscopy-assisted distal gastrectomy for early gastric cancer: results of a prospective randomized clinical trial. Ann Surg 2008;248:721-7.
11. Hayashi H, Ochiai T, Shimada H, Gunji Y. Prospective randomized study of open versus laparoscopy-assisted distal gastrectomy with extraperigastric lymph node dissection for early gastric cancer. Surg Endosc 2005;19:1172-6.

12. Kim HH, Hyung WJ, Cho GS, Kim MC, Han SU, Kim W, et al. Morbidity and mortality of laparoscopic gastrectomy versus open gastrectomy for gastric cancer: an interim report-a phase III multicenter, prospective, randomized trial (KLASS Trial). Ann Surg 2010;251:417-20.

13. Lee JH, Kim YW, Ryu KW, Lee JR, Kim CG, Choi IJ, et al. A phase-II clinical trial of laparoscopy-assisted distal gastrectomy with D2 lymph node dissection for gastric cancer patients. Ann Surg Oncol 2007;14:3148-53.

14. Fujiwara M, Kodera Y, Kasai Y, Kanyama Y, Hibi K, Ito K, et al. Laparoscopy-assisted distal gastrectomy with systemic lymph node dissection for early gastric carcinoma: a review of 43 cases. J Am Coll Surg 2003;196:75-81.

15. Kitano S, Shiraishi N, Uyama I, Sugihara K, Tanigawa N. A multicenter study on oncologic outcome of laparoscopic gastrectomy for early cancer in Japan. Ann Surg 2007;245:68-72.

16. Huscher CG, Mingoli A, Sgarzini G, Sansonetti A, Di Paola M, Recher A, et al. Laparoscopic versus open subtotal gastrectomy for distal gastric cancer: 5-year results of a randomized prospective trial. Ann Surg 2005;241:232-7.

17. Japanese Gastric Cancer Association. Japanese classification of gastric carcinoma. - 2nd English edition - Gastric Cancer 1998:1:10-24.

18. JCOG Surgical Committee. JCOG surgical morbidity criteria, 2005 (in Japanese). http://wwwjcogjp/doctor/tool/C_150_0021pdf. Accessed 22 Apr 2010.

19. Hori S, Ochiai T, Gunji Y, Hayashi H, Suzuki T. A prospective randomized trial of hand-sutured versus mechanically stapled anastomoses for gastroduodenostomy after distal gastrectomy. Gastric Cancer 2004;7:24-30.

20. Sasako M, Katai H, Sano T, Maruyama K. Management of complications after gastrectomy with extended lymphadenectomy. Surg Oncol 2000;9:31-4.

21. Inomata M, Yasuda K, Shiraishi N, Kitano S. Clinical evidences of laparoscopic versus open surgery for colorectal cancer. Jpn J Clin Oncol 2009;39:471-7.

22. Strong VE, Devaud N, Allen PJ, Gonen M, Brennan MF, Coit D. Laparoscopic versus open subtotal gastrectomy for adenocarcinoma: a case-control study. Ann Surg Oncol 2009;16:1507-13.

23. Pugliese R, Maggioni D, Sansonna F, Costanzi A, Ferrari GC, Di Lernia S, et al. Subtotal gastrectomy with $\mathrm{D} 2$ dissection by minimally invasive surgery for distal adenocarcinoma of the stomach: results and 5-year survival. Surg Endosc 2010. doi: 10.1007/ s00464-010-1014-1.

24. Kubo M, Sano T, Fukagawa T, Katai H, Sasako M. Increasing body mass index in Japanese patients with gastric cancer. Gastric Cancer 2005;8:39-41.

25. Mochiki E, Kamiyama Y, Aihara R, Nakabayashi T, Asao T, Kuwano H. Laparoscopic assisted distal gastrectomy for early gastric cancer: 5 years' experience. Surgery 2005;137:317-22.

26. Bone RC, Balk RA, Cerra FB, Dellinger RP, Fein AM, Knaus WA, et al. Definitions for sepsis and organ failure and guidelines for the use of innovative therapies in sepsis. The ACCP/SCCM Consensus Conference Committee. American College of Chest Physicians/Society of Critical Care Medicine. Chest 1992;101:1644-55.

27. Adachi Y, Shiraishi N, Shiromizu A, Bandoh T, Aramaki M, Kitano S. Laparoscopy-assisted Billroth I gastrectomy compared with conventional open gastrectomy. Arch Surg 2000;135:806-10.

28. Kawamura H, Okada K, Isizu H, Masuko H, Yamagami H, Honma $\mathrm{S}$, et al. Laparoscopic gastrectomy for early gastric cancer targeting as a less invasive procedure. Surg Endosc 2008;22:81-5. 